\title{
Modeling and seismic migration in anisotropic media as a function of azimuthal angle - $\mathrm{HTI}(\Psi)$
}

\begin{abstract}
In this paper, we present a new way of modeling in an anisotropic medium based on a pseudo-acoustic one-way equation, derived from the full equations system of elasticity using eigenvalues of dispersion equation - time-frequency. The method was shown on the examples of signals propagation in anisotropic medium HTI( $($ ) (Horizontal Transverse Isotropy) as function of azimuthal angle $\Psi$ and zero-offset time sections for anticline model. The correctness of the modeling results was verified by new migration $\mathrm{MG}(\mathrm{F}-\mathrm{K})$ in wavenumbers $(\mathrm{K})$ - frequency $(\mathrm{F})$ domain with a depth operator of extrapolation, which uses a vertical wavenumber derived from dispersion relation.
\end{abstract}

Key words: Transverse Isotropy (TI), Horizontal Transverse Isotropy (HTI), dispersion relation, migration in wavenumber-frequency domain MG(F-K), Thomsen's parameters, azimuthal migration.

\section{Modelowanie i migracja sejsmiczna w anizotropowym ośrodku HTI( $\Psi)$ w funkcji kąta azymutalnego $\Psi$}

W artykule przedstawiono nowy sposób modelowania w ośrodkach anizotropowych, bazujący na jednostronnym równaniu falowym wyprowadzonym z pełnego systemu równań sprężystości i stosującym wartości własne równania dyspersyjnego tj. czasową częstotliwość. Metoda została zaprezentowana na przykładach propagacji w ośrodkach

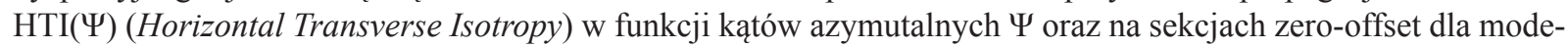
lu antykliny. Poprawność rezultatów modelowania została zweryfikowana za pośrednictwem migracji MG(F-K) w dziedzinie liczb falowych (K) i częstotliwości (F) stosującej operator głębokościowej ekstrapolacji z użyciem pionowej liczby falowej wyprowadzonej z relacji dyspersyjnej.

Słowa kluczowe: poprzeczna izotropia, poziomo-poprzeczna izotropia, relacja dyspersyjna, migracja MG(F-K) w dziedzinie liczb falowych i częstotliwości, parametry Thomsena, migracja azymutalna.

\section{Introduction}

Anisotropic media of HTI type (Horizontal Transverse Isotropy) (Fig. 1) represented by the system of vertical, parallel isotropic planes are substantial elements of deep geologic structures and facial sequences, which in practice determines the necessary condition to determine the direction of cracks in shale rocks by the seismic investigations. That means, the velocity of compressional wave in isotropy plane i.e. in vertical plane, differs from the velocity in perpendicular direction to isotropy plane and it can constitute the indicator of the cracks orientations [29].

The imaging of geological structures in this case, requires knowledge of velocity as a function of azimuthal angle

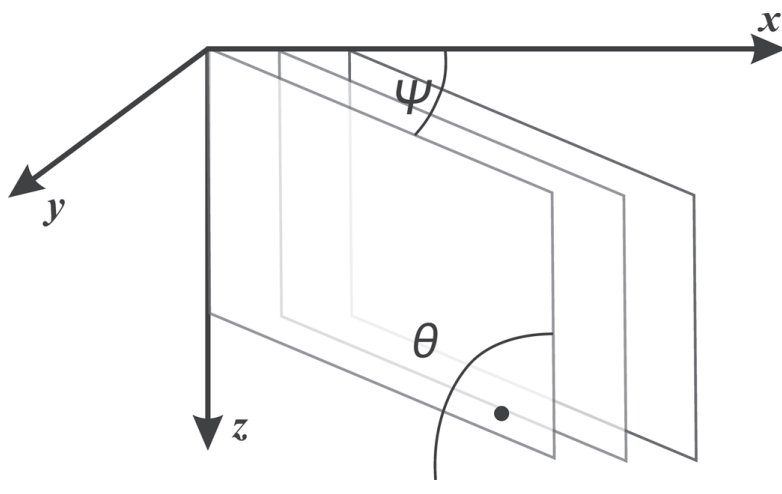

Fig. 1. Draft presents a HTI media - $\theta$ indicates a dip angle, $\Psi$ - an azimuthal angle 
between the strike of isotropy plane (plane of cracks) and the direction of measurements line and an application of migration which takes into consideration a real run time of wave in media. Thus, the task in reality is reduced to solving the question of compressional waves propagation in anisotropic media $\operatorname{HTI}(\Psi)$ as a function of azimuthal angle $\Psi$.

\section{Theory in outline}

In general, forward modeling of wavefields in anisotropic media should be executed by solving the full system of elastic equations determined by Hooke's law and equations of motion. It follows from this, that each component of stress tensor $T_{i j}$ is a linear function of strain $E_{l k}$ :

$$
\begin{aligned}
& T_{i j}=D^{\theta, \Psi} E_{k l} \\
& \text { and } \\
& E_{l k}=1 / 2\left(U_{l, k}+U_{k, l}\right)
\end{aligned}
$$

where $U_{l, k}=\frac{\partial U_{l}}{\partial k}$ is a derivative of $l$ component of displacement with respect to the $k$-th coordinate.

In relation (1) stiffness tensor is shown in the form of symmetrical matrix $D^{\theta, \Psi}(6 \times 6)$, whose components are functions of dip angle $\theta$, angle $\Psi$ between strike and measurement line, velocities of compressional and shear waves, and anisotropy parameters. Tensor $D^{\theta, \Psi}$, received as the result of the transformation of tensor $C$, characterizes VTI (Vertical Transverse Isotropy) media with vertical symmetry according to Bond's relation [4, 32].

Using the equations of motion we have:

$$
\begin{aligned}
& T_{11,1}+T_{12,2}+T_{13,3}=\rho \frac{\partial^{2} U_{1}}{\partial t^{2}} \\
& T_{21,1}+T_{22,2}+T_{23,3}=\rho \frac{\partial^{2} U_{2}}{\partial t^{2}} \\
& T_{31,1}+T_{32,2}+T_{33,3}=\rho \frac{\partial^{2} U_{3}}{\partial t^{2}}
\end{aligned}
$$

where $\rho$ is density of medium and subscript " $i j, j$ " indicates the partial derivative with respect to the $j$-th coordinate.

The equations (2) constitute the full system of second order equations with respect to time, its solution is a very laborious computation procedure. Therefore, the tendency arose to simplify the way of computation. Alkhalifah $[1,2]$ has introduced into practice pseudo-acoustic fourth order equations in space coordinates which for the VTI model proved to be a promising approximation and an inspiration for other modifications [9, 10, 12, 31].

The essential assumption for this type of approximation is the acceptance, that the velocity of a shear wave is zero $V_{S V}=0$. This assumption is justified by negligible influence of the velocity $V_{S V}$ on the phase velocity of the compressional wave $[1,2]$.

For HTI( () model we will use the same approach that was applied to wave propagation in transverse isotropy media TI (VTI, TTI) [17, 18].

This approach relies on the determination of the eigenvalue - time-frequency $\omega_{a}$ from matrix dispersion relation:

$$
\left[\begin{array}{lll}
a_{11} & a_{12} & a_{13} \\
a_{21} & a_{22} & a_{23} \\
a_{31} & a_{32} & a_{33}
\end{array}\right]\left[\begin{array}{l}
U_{x} \\
U_{y} \\
U_{z}
\end{array}\right]=0
$$

that is obtained as a result of Fourier transform application $\left(x \rightarrow k_{x}, y \rightarrow k_{y}, z \rightarrow k_{z}, t \rightarrow \omega_{a}\right)$ to the system of equation (2), where:

$$
\begin{aligned}
& a_{11}=d_{11} k_{x}^{2}+d_{66} k_{y}^{2}+d_{55} k_{z}^{2}+2 d_{16} k_{x} k_{y}-\omega_{a}^{2} \\
& a_{12}=a_{21}=d_{16} k_{x}^{2}+d_{26} k_{y}^{2}+d_{54} k_{z}^{2}+\left(d_{12}+d_{66}\right) k_{y} k_{x} \\
& a_{13}=a_{31}=\left(d_{55}+d_{13}\right) k_{x} k_{z}+\left(d_{45}+d_{36}\right) k_{y} k_{z} \\
& a_{22}=d_{66} k_{x}^{2}+d_{22} k_{y}^{2}+d_{44} k_{z}^{2}+2 d_{26} k_{x} k_{y}-\omega_{a}^{2} \\
& a_{23}=a_{32}=\left(d_{63}+d_{45}\right) k_{x} k_{z}+\left(d_{23}+d_{44}\right) k_{y} k_{z} \\
& a_{33}=d_{55} k_{x}^{2}+d_{44} k_{y}^{2}+d_{33} k_{z}^{2}+2 d_{45} k_{x} k_{y}-\omega_{a}^{2}
\end{aligned}
$$

and $k_{x}, k_{\mathrm{y}}, k_{z}$ are components of wave vector expressed in wavenumbers.

Solution of equation (3) requires the compliance of conditions:

$$
\operatorname{det} A=\operatorname{det}\left[\begin{array}{lll}
a_{11} & a_{12} & a_{13} \\
a_{21} & a_{22} & a_{23} \\
a_{31} & a_{32} & a_{33}
\end{array}\right]=0
$$

where det means determinant of matrix $A$.

The determinant is expressed by relation:

$$
\operatorname{det} A=H\left(k_{x}, k_{y}, k_{z}, \theta=90^{\circ}, \Psi\right)-\omega_{a}^{6}+\omega_{a}^{4} B-\omega_{a}^{2} C=0
$$

For a 2D case, when $k_{y}=0$, and for acoustic model of propagation, i.e. for velocity of shear wave $V_{S V}=0$, we receive relation:

$$
\begin{aligned}
& H\left(k_{X}, k_{z}, \theta=90^{\circ}, \Psi\right)=k_{X}^{4} k_{z}^{2}\left[\begin{array}{l}
d_{11}\left(d_{33} d_{66}+d_{63}^{2}\right)-d_{16}^{2} d_{33}+ \\
+d_{13} d_{16} d_{66}-d_{13}^{2} d_{66}
\end{array}\right] \\
& B=k_{X}^{2}\left(d_{11}+d_{66}\right)+k_{z}^{2} d_{33} \\
& C=k_{X}^{2} k_{z}^{2}\left(d_{11} d_{33}+d_{66} d_{33}-d_{63}^{2}-d_{13}^{2}\right)+k_{X}^{4}\left(d_{11} d_{66}-d_{16}^{2}\right)
\end{aligned}
$$

where $d_{i j}$ are components of stiffness tensor $D^{\theta, \Psi}$ (see Appendix).

In range of variability of the parameters $\varepsilon(0.3 ;-0.2)$ and 
$\delta(0.2 ;-0.2)$ one can allow that function $H \approx 0$ and then from relation (6) we obtain biquadratic polynomial:

$\omega_{a}^{4}-\omega_{a}^{2}\left[k_{x}^{2}\left(d_{11}+d_{66}\right)+k_{z}^{2} d_{33}\right]+k_{x}^{2} k_{z}^{2}\left[d_{11} d_{33}+d_{66} d_{33}-d_{63}^{2}-d_{13}^{2}\right]+$

$+k_{x}^{4}\left[d_{11} d_{66}-d_{16}^{2}\right]=0$

Furthermore, the elimination of components $d_{66}, d_{63}, d_{16}$ from (8) leads to equation:

$$
\omega_{a}^{4}-\omega_{a}^{2}\left(d_{11} k_{x}^{2}+d_{33} k_{z}^{2}\right)+k_{x}^{2} k_{z}^{2}\left\lfloor d_{11} d_{33}-d_{13}^{2}\right\rfloor=0
$$

From relation (6) (or equivalent third order Cardano's equation) and from biquadratic equations (8-9) and also from biquadratic equations in VTI and TTI media (Tilted Transverse Isotropy) [18] it follows this, that $\omega_{a}$ as eigenvalue of dispersion relations is expressed as follows:

$$
\omega_{a}=V_{p} \cdot k_{a}
$$

where $V_{p}$ is the velocity of a compressional wave in a direction along symmetry axis and $k_{a}=k_{a}\left(k_{x}, k_{z}, \theta, \Psi, \varepsilon, \delta\right)$ is a function of anisotropy parameters $\varepsilon, \delta$, the components of wave vector $k=\left(k_{x}^{2}+k_{z}^{2}\right)^{1 / 2}$ and angles: $\theta$ and $\Psi$.

The relation (10), the result of velocity separation $V_{p}$ as solutions for eigenvalue, has a universal character for TI models class. Through multiplication of both sides of the equation (10) by scalar field $P\left(k_{x}, k_{z}, \omega_{a}\right)$ in wavenumber and frequency domain and application of Fourier transform $\left(\omega_{a} \rightarrow t\right)$ we obtain:

$$
\frac{\partial P\left(k_{x}, k_{z}, t\right)}{\partial t}= \pm i V_{p} k_{a} P\left(k_{x}, k_{z}, t\right)
$$

i.e. one-way wave equation, or in two-side version

$$
\frac{1}{V_{p}^{2}} \frac{\partial^{2} P\left(k_{x}, k_{z}, t\right)}{\partial t^{2}}=-k_{a}^{2} P\left(k_{x}, k_{z}, t\right)
$$

called pseudo-acoustic equations.

General solution of equation (12) is the relation expressed in Fourier transform $\left(k_{x} \rightarrow x, k_{z} \rightarrow z\right)$

$$
P(x, z, t)=\sum_{k x} \sum_{k z} P\left(k_{x}, k_{z}, t=0\right) \exp \left[i\left(k_{x} x+k_{z} z \pm \omega_{a} t\right)\right]
$$

In inhomogeneous media equation (11) should be replaced by pseudo-spectral form:

$$
\frac{\partial P(x, z, t)}{\partial t}=i \mathrm{~V}_{\mathrm{p}} F^{-1}\left\{k_{a} F[P(x, z, t)]\right\}
$$

where $F\left(x \rightarrow k_{x}, z \rightarrow k_{z}\right)$ and $F^{-1}\left(k_{x} \rightarrow x, k_{z} \rightarrow z\right)$ are operators of Fourier transform from $(x, y, z)$ domain to $\left(k_{x}, k_{y}, k_{z}\right)$ domain and vice versa.

Relation (13) for $t=0$, i.e. $P(x, z, t=0)$ is initial conditions for one-way equation (14).

Numerical solution of equation (14) can be obtained by means of the Taylor's series limited to third term

$$
P(x, z, t)=\sum_{l=0}^{3} \frac{\partial^{l} P(x, z, 0)}{\partial t^{l}} \frac{\Delta t^{l}}{l !}
$$

In isotropic case, I. Gazdag [14] proved stability of scheme (15). In general case, referred to equation (9) the stability demands the fulfillment of the condition:

$$
\begin{aligned}
& q>0 \\
& 1+2 \delta>0
\end{aligned}
$$

and

$$
\left\{\begin{array}{l}
q\left[q \cos ^{4} \Psi+\sin ^{4} \Psi+2(1+2 \delta)^{1 / 2} \sin ^{2} \Psi \cos ^{2} \Psi\right]- \\
-2 \eta_{a} \sin ^{4} \Psi
\end{array}\right\}>0
$$

where $q=1+2 \varepsilon, \eta_{a}=2(\varepsilon-\delta), \varepsilon, \delta$ - Thomsen's parameters.

The stability of numerical solution with method employing the differencing schema is satisfied as for "isotropy" case [14], i.e.

$$
\omega_{a} \Delta t \leq 2
$$

where $\Delta t$ is time increment. In practice step $\Delta t=0.5 \mathrm{~ms}$ seems to be the reasonable compromise between the stability and the accuracy of the method.

\section{Migration}

In media of the TI type most frequently Kirchhoff's migration is used in practice, because of the relative simplicity of this method. The Kirchhoff's migration requires multipatching of seismic rays $[8,13,26,27,28]$ or application of the finite difference method [3]. Strong contrasts of velocity lead to some problems for both methods, which can create significant errors of depth imaging.

Podwin P. and Lecompte I. [25] proposed algorithm for strong heterogeneous media in the case that the wavefront is approximated by plane wave well. In monoclinal media with inclined axis of symmetry TTI Kumar D., Sen M., Ferguson R. [22] developed algorithm by Faria and Stoffa [11] taking into account anisotropy parameters as functions of space coordinates. Reverse time migration takes special position for the effectiveness of imaging structures [5, 10, 12, 24, 30]. For TI type media algorithms were worked-out for VTI and TTI models.

In this paper we present $\mathrm{MG}(\mathrm{F}-\mathrm{K})$ migration which will be adapted to the imaging of 2D anisotropic media of the HTI( $\Psi)$ type. This migration was created for compressional waves in pre-stack version $[16,19,20]$ and in post-stack version [19]. 


\section{Basic equation for wavefields depth extrapolation}

The starting point for the migration process is the depth extrapolation of the wavefield $U(x, z, \omega)$ from level $z_{j}$ to level $z_{j}+\Delta z$

$$
U_{z_{j}+\Delta z}=F_{j}(x, \omega) U^{\prime}\left(x, z_{j}+\Delta z, \omega\right)
$$

where $U^{\prime}\left(x, z_{j}+\Delta z, \omega\right)$ is Fourier transform $\left(k_{x} \rightarrow x\right)$ of the wavefield

$$
U^{\prime}\left(k_{x}, z_{j}+\Delta z, \omega\right)=e^{-i k_{z 0}^{H T I} \Delta z} U\left(k_{x}, z_{j}, \omega\right)
$$

and where: $x$ is the horizontal coordinate along the measurement profile, $j$ indicates subsequent depth interval $z_{j+1}-z_{j}=\Delta z$, $k_{z_{0}}^{H T I}$ denotes vertical wavenumber in homogenous media HTI( $\left.\Psi\right)$.

Relation

$$
F_{j}(x, \Delta z, \omega)=\left[1+\frac{i \omega^{2}}{2} \cdot \Delta z \cdot M_{j}(x, \omega)\right]^{-1}
$$

determinates the correction of the wavefield and is the sum of Neumann's series, and $M_{j}(x, \omega)$ denotes

$$
M_{j}(x, \omega)=\sum_{k_{x}}\left(k_{z_{0}}^{H T I}\right)^{-1}\left[\left(k_{z_{0}}^{H T I}\right)^{2}-\left(k_{z}^{H T I}\right)^{2}\right] e^{i k_{x} x} d k_{x}
$$

where $k_{z}^{H T I}$ is vertical wavenumber in heterogeneous media, i.e. where velocity $V_{p}$ and Thomsen's parameters $\varepsilon$ and $\delta$ are functions of $x$ space coordinate.

It follows from relations (17-19) that this migration has two stages. In first stage, the extrapolation is made in homogeneous media for constant velocity $V_{p}$ and parameters $\varepsilon$ and $\delta$ in range of measurement interval. From practical point of view it is convenient to use average values in the interval of measurement. In second stage the correction of wavefield proceeds by function $F_{j}(x, \omega)$.

The stability for down extrapolation process succeeds through the convergence of Neumann's series:

$$
\left[1+\frac{i \omega^{2} \Delta z}{2} \cdot M_{j}(x, \omega)\right]^{-1}
$$

what is done if condition

$$
\left|\frac{i \omega^{2} \Delta z}{2} \cdot M_{j}(x, \omega)\right|<1
$$

is fulfilled, which finally assures stability of extrapolation.

\section{Pre-stack migration}

The equations (17-20) should be treated as the starting point for the depth extrapolation process before stacking of wavefield exited by many receivers, deployed along the profile. Imaging of the depth target is finished when the coincidence of sources and receivers position is fulfilled in time $t=0$. It means that sources and receivers should be displaced in depth, until they reach the time-space coincidence. In the rubric of wave equations that means the alternate use of extrapolation vs. source and receivers.

Denoting the coordinates of the sources positions by $s$ and receivers by $g$ we obtain for extrapolation the following relation:

$$
\begin{aligned}
& U_{j}\left(g, s, z_{j}+\Delta z, \omega\right)= \\
& =F_{j}(g, \Delta z, \omega) F_{j}(s, \Delta z, \omega) U^{\prime}{ }_{j}\left(g, s, z_{j}+\Delta z, \omega\right)
\end{aligned}
$$

where $U_{j}^{\prime}\left(g, s, z_{j}+\Delta z, \omega\right)$ is double Fourier transform $\left(k_{g} \rightarrow g\right.$, $\left.k_{s} \rightarrow s\right)$ of wavefield:

$U^{\prime}{ }_{j}\left(k_{g}, k_{s}, z_{j}+\Delta z, \omega\right)=U_{j}\left(k_{g}, k_{s}, z_{j}, \omega\right) e^{-i\left(k_{z_{g}}^{H I T}+k_{z_{s}}^{H T I}\right) \Delta z}$

in other words the function of the horizontal wavenumber $k_{g}$ and $k_{s}$, which corresponds to space coordinates of sources $s$ and receivers $g$.

Correction filters $F_{j}(g, \Delta z, \omega)$ and $F_{j}(s, \Delta z, \omega)$ relating to receivers and sources are expressed by analogical relations as (19) and (20) with the difference that $x$ coordinate should be substituted by $s$ and $g$ coordinates. These coordinates define slowness $S$ and Thomsen's parameters in the expression of vertical wavenumbers $k_{z_{s}}^{H T I}$ and $k_{z_{g}}^{H T I}$.

It is convenient to use common reference values of vertical wavenumber in relations for $M_{j}(s)$ and $M_{j}(g)$.

The extrapolation procedure under relations (23-24) requires the application of double Fourier transformation ( $g \rightarrow k_{g}, s \rightarrow k_{s}$ ) to the wavefield in order to shift receivers and sources on subsequent level $z_{j}$ of depth. Imaging of depth structures is done at point of coincidence for sources and receivers when $s=g$ for time $t=0$ :

$$
U_{j_{\text {migr. }}}\left(s=g, z_{j}+\Delta z, t=0\right)=\sum_{\omega} U_{j}\left(s=g, z_{j}+\Delta z, \omega\right) d \omega
$$

where $U_{j_{\text {migr }}}$ stands for wavefield at depth $z_{j}+\Delta z$.

The stability of the extrapolation algorithm requires the convergence of Neumann series analogously to relation (21). 


\section{Zero-offset migration}

In case of zero-offset migration one assumes that $x$ coordinate on the profile is the coordinate of source as well as the receiver. The wavefield at registration level $z=0$ one can obtain in the process of modeling, provided that at each point of the velocity discontinuity wave is simultaneously excited at time $t=0$ and propagates into $z=0$ level, with velocity equal $1 / 2$ of real velocity [23]. Therefore in the equations describing waves propagation, $2 S$ slowness should be used instead of $S$. It should be noted that the approximation of the zero-offset section is in practice the stacked time section.

In case of zero-offset migration, the depth extrapolation formulated by (17) will be expressed as follows:

$$
U_{z_{j}+\Delta z}=\left[1+i 2 \omega^{2} \Delta z M_{j}(x, \omega)\right]^{-1} U^{\prime}\left(x, z_{j}+\Delta z, \omega\right)
$$

where $U^{\prime}\left(x, z_{j}+\Delta z, \omega\right)$ is defined by relation (18).

Imaging of depth structures is obtained as a result of Fourier transform $(\omega \rightarrow t)$ for $t=0$ time:

$$
U_{\text {migr }}\left(x, z_{j}+\Delta z, t=0\right)=\sum_{\omega} U\left(x, z_{j}+\Delta z, \omega\right)
$$

which leads to the summation of the wavefield for a useful range of frequency.

\section{Depth extrapolation operators}

Vertical wavenumbers $k_{z_{s}}^{H T I}$ and $k_{z_{g}}^{H T I}$ are solutions of dispersion relation (6), they can be expressed as follows:

$k_{z}^{H T I}=\left\{\frac{\omega^{6}-\omega^{4} k_{x}^{2}\left(d_{11}+d_{66}\right)+\omega^{2} k_{x}^{4}\left(d_{11} d_{66}-d_{16}^{2}\right)}{\omega^{4} d_{33}-\omega^{2} k_{x}^{2} N_{1}+k_{x}^{4} N_{2}}\right\}^{1 / 2}$

where

$$
\begin{aligned}
& N_{1}=d_{33}\left(d_{11}+d_{66}\right)-d_{63}{ }^{2}-d_{13}{ }^{2} \\
& N_{2}=d_{11}\left[d_{33} d_{66}-d_{63}^{2}\right]-d_{16}^{2} d_{33}+d_{13} d_{16} d_{63}-d_{13}^{2} d_{66}
\end{aligned}
$$

Neglecting $d_{66}, d_{63}, d_{16}$ components of elasticity tensor $\mathrm{D}^{90^{\circ} . \Psi}$ as small values in comparison to $d_{11}, d_{33}, d_{13}$ we have approximation

$k_{z}^{H T I}=\left\{\frac{\omega^{4}-\omega^{2} k_{x}^{2} d_{11}}{\omega^{2} d_{33}-k_{x}^{2}\left(d_{11} d_{33}-d_{13}{ }^{2}\right)}\right\}^{1 / 2}=$

$=\left\{\frac{\omega^{4} S^{4}-\omega^{2} S^{2} k_{x}^{2}\left[(1+2 \varepsilon) \cos ^{4} \Psi+\sin ^{4} \Psi+1 / 2(1+\delta) \sin ^{2}(2 \Psi)\right]}{\omega^{2} S^{2}(1+2 \varepsilon)-k_{x}^{2} \sin ^{4} \Psi \cdot \eta_{a}}\right\}^{1 / 2}$

where $S=V_{p}^{-1} ; \eta_{a}=2(\varepsilon-\delta)$.

\section{Numerical experiments}

\section{Modeling of signals propagation}

A series of signals propagation modeling of "spike" type wavelet in anisotropic media with parameters: $\varepsilon=0.3$; $\delta=-0.1, \Psi=0^{\circ}, 30^{\circ}, 45^{\circ}, 60^{\circ}, 90^{\circ}, 120^{\circ}$ for azimuthal angles and $V_{P}=2000 \mathrm{~m} / \mathrm{s}$ for velocity was performed (Fig. 2-7).

Snapshots showing waves excited at point source $(0.0)$, present the moment of time $t=0.4 \mathrm{~s}$. The exact algorithm of propagation (6) was applied. In general, the series of "spike" type propagating waves in the $X Z$ plane shows their correctness and high precision. Visual estimation indicates the convergence of propagation ways with assumptions, numerically it shows the error $\sim 0.2 \%$ for time $t=0.4 \mathrm{~s}$.

All images for azimuthal angle range $0^{\circ}-120^{\circ}$ are devoid of the "diamond shape" type noise, so characteristic for propagation described by pseudo-acoustic equations in space coordinates [7]. In the computations, one-way equation (11) was applied, then two image versions, "upward" and "downward" were stuck together and shown as one. Visible discontinuities of signals in some images are caused by the presentation of every fourth trace, which leads to the loss of signal coincidence at places of flatness of wavefront. This occurrence is random.

Figures 8-9 exhibit a composition of images of wave propagation in media with parameters $\varepsilon=0.3$ and $\delta=0.1$ for angles $\Psi=0^{\circ}, 15^{\circ}, 30^{\circ}, 45^{\circ}, 60^{\circ}, 75^{\circ}, 90^{\circ}$ in the $X Z$ plane. Here we can observe a velocity decrease with an increase of azimuthal angle along the $X$ axis. For angle $\Psi=90^{\circ}$ the velocity achieves minimum value, i.e. assuming $V_{P}=2000 \mathrm{~m} / \mathrm{s}$ in a perpendicular direction to the symmetry plane, whereas in the direction along the $Z$ axis propagation velocity is $V=V_{p}(1+2 \varepsilon)^{1 / 2}=2529 \mathrm{~m} / \mathrm{s}$. From that we can easily note that velocity as function of the azimuthal angle has an ellipsoidal character and is expressed as follows:

$$
V_{P}(\Psi)=V_{P}\left(1+2 \varepsilon \cos ^{2} \Psi\right)^{1 / 2}
$$

This relation indirectly confirms the correctness of dependence velocity $V_{N M O}$ as a function of the azimuthal angle $\Psi$ derived by W. Grechka and I. Tsvankin [15] for 3D dimensions. 


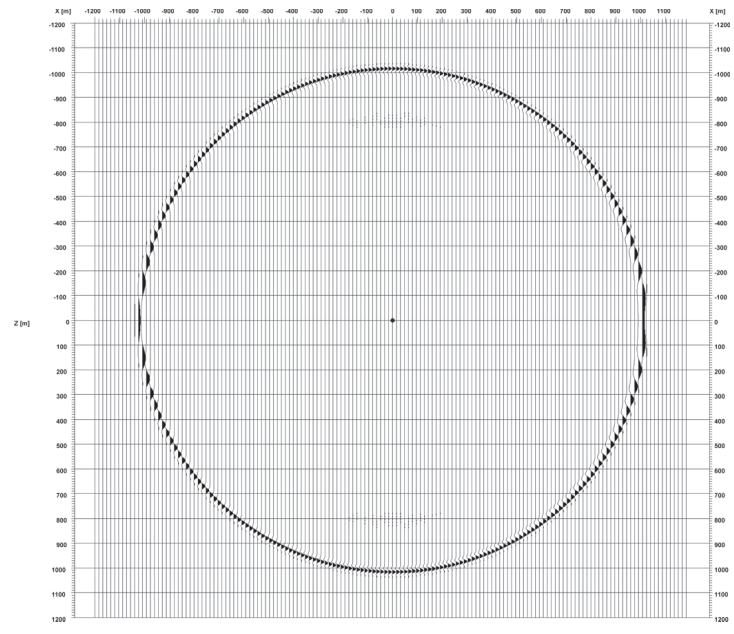

Fig. 2. Snapshot of spike signal propagation from relation (6) in moment time $t=0.4 \mathrm{~s}$; anisotropy parameter's: $\varepsilon=0.3$; $\delta=-0.1$; azimuthal angle $\Psi=0^{\circ}$; steps of computations:

$$
\Delta x=\Delta z=4 \mathrm{~m}, \Delta t=0.5 \mathrm{~ms}
$$

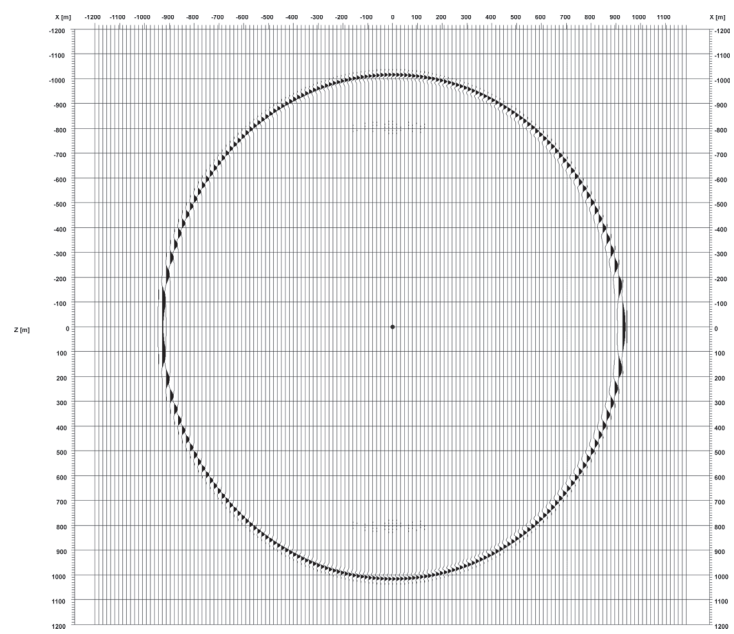

Fig. 3. Snapshot of spike signal propagation from relation (6) in moment time $t=0.4 \mathrm{~s}$; anisotropy parameter's: $\varepsilon=0.3$; $\delta=-0.1$; azimuthal angle $\Psi=30^{\circ}$; steps of computations: $\Delta x=\Delta z=4 \mathrm{~m}, \Delta t=0.5 \mathrm{~ms}$

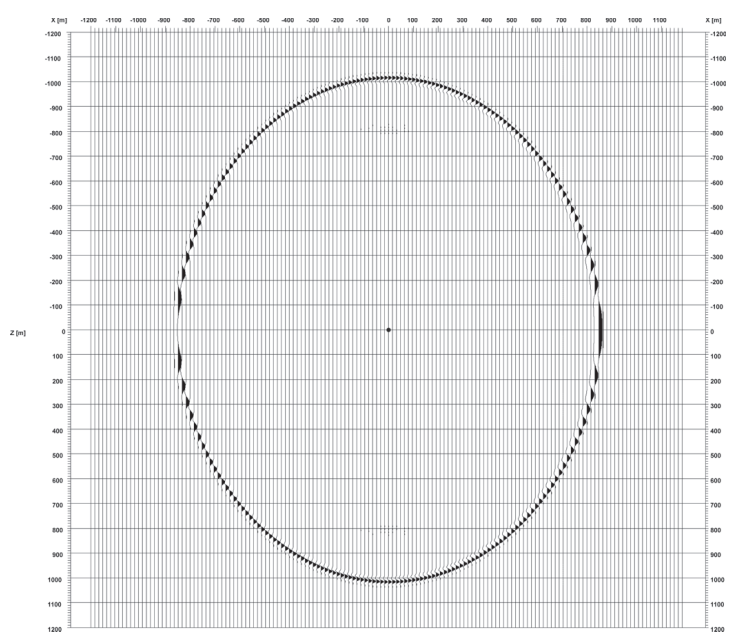

Fig. 4. Snapshot of spike signal propagation from relation (6) in moment time $t=0.4 \mathrm{~s}$; anisotropy parameter's: $\varepsilon=0.3$; $\delta=-0.1$; azimuthal angle $\Psi=45^{\circ}$; steps of computations: $\Delta x=\Delta z=4 \mathrm{~m}, \Delta t=0.5 \mathrm{~ms}$

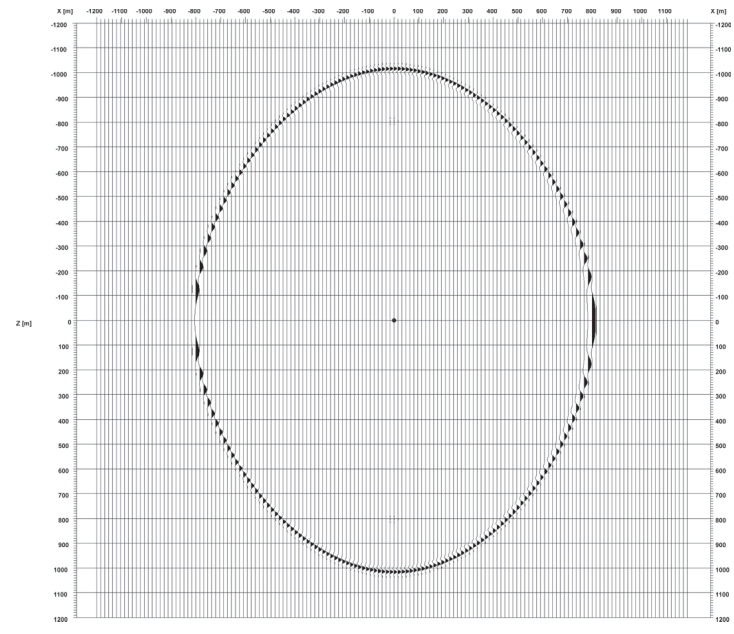

Fig. 5. Snapshot of spike signal propagation from relation (6) in moment time $t=0.4 \mathrm{~s}$; anisotropy parameter's: $\varepsilon=0.3$; $\delta=-0.1$; azimuthal angle $\Psi=60^{\circ}$; steps of computations: $\Delta x=\Delta z=4 \mathrm{~m}, \Delta t=0.5 \mathrm{~ms}$

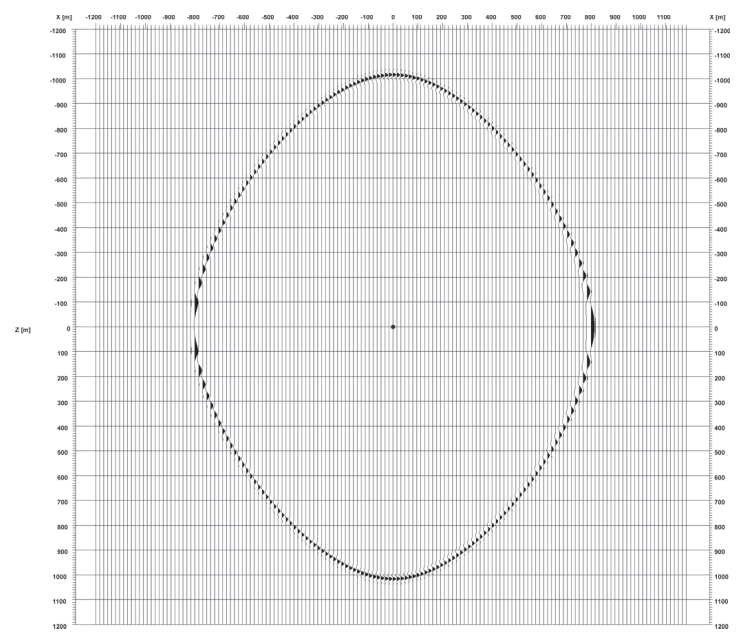

Fig. 6. Snapshot of spike signal propagation from relation (6) in moment time $t=0.4 \mathrm{~s}$; anisotropy parameter's: $\varepsilon=0.3$; $\delta=-0.1$; azimuthal angle $\Psi=90^{\circ}$; steps of computations: $\Delta x=\Delta z=4 \mathrm{~m}, \Delta t=0.5 \mathrm{~ms}$

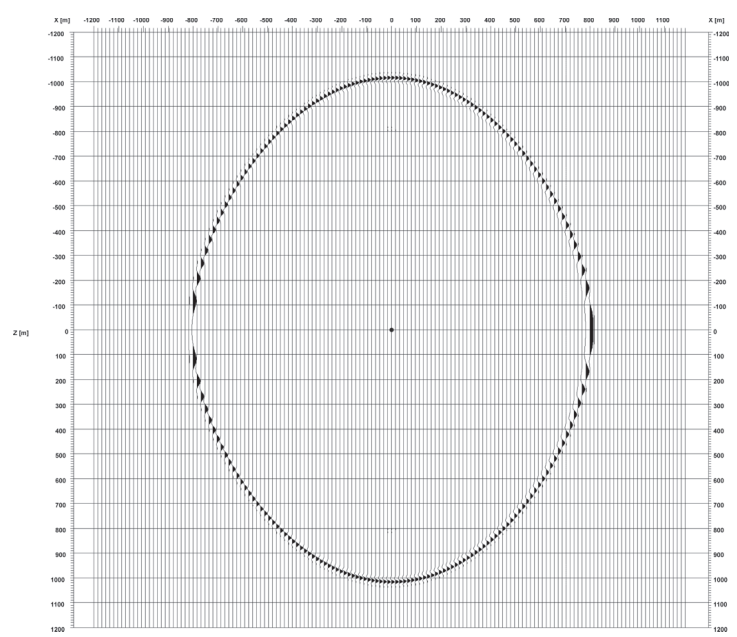

Fig. 7. Snapshot of spike signal propagation from relation (6) in moment time $t=0.4 \mathrm{~s}$; anisotropy parameter's: $\varepsilon=0.3$; $\delta=-0.1$; azimuthal angle $\Psi=120^{\circ}$; steps of computations: $\Delta x=\Delta z=4 \mathrm{~m}, \Delta t=0.5 \mathrm{~ms}$ 


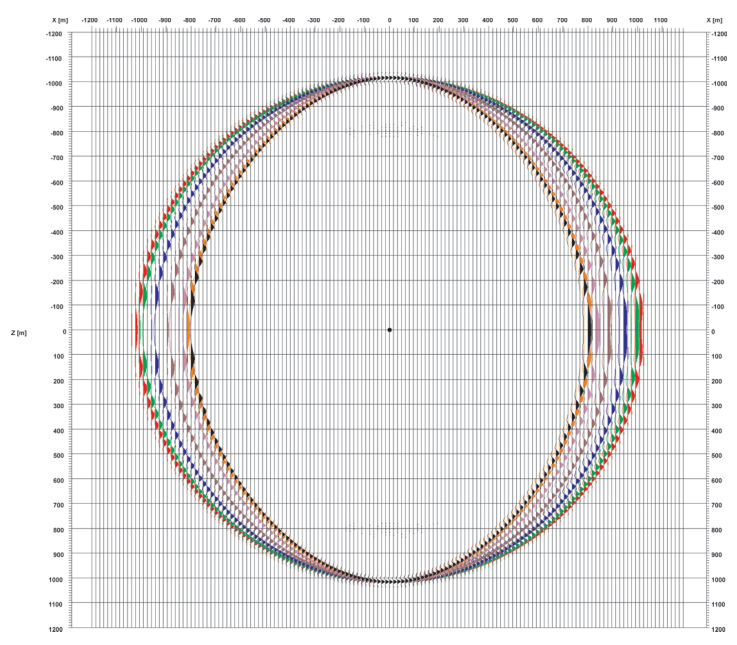

Fig. 8. Comparison of the snapshot of propagation from (6) in moment time $t=0.4 \mathrm{~s}$; anisotropy parameters: $\varepsilon=0.3 ; \delta=0.1$; for azimuthal angle: $\Psi=0^{\circ}-$ in red, $\Psi=15^{\circ}-$ in green, $\Psi=30^{\circ}-$ in blue, $\Psi=45^{\circ}$ - in crimson, $\Psi=60^{\circ}$ - in violet, $\Psi=75^{\circ}-$ in orange, $\Psi=90^{\circ}-$ in black

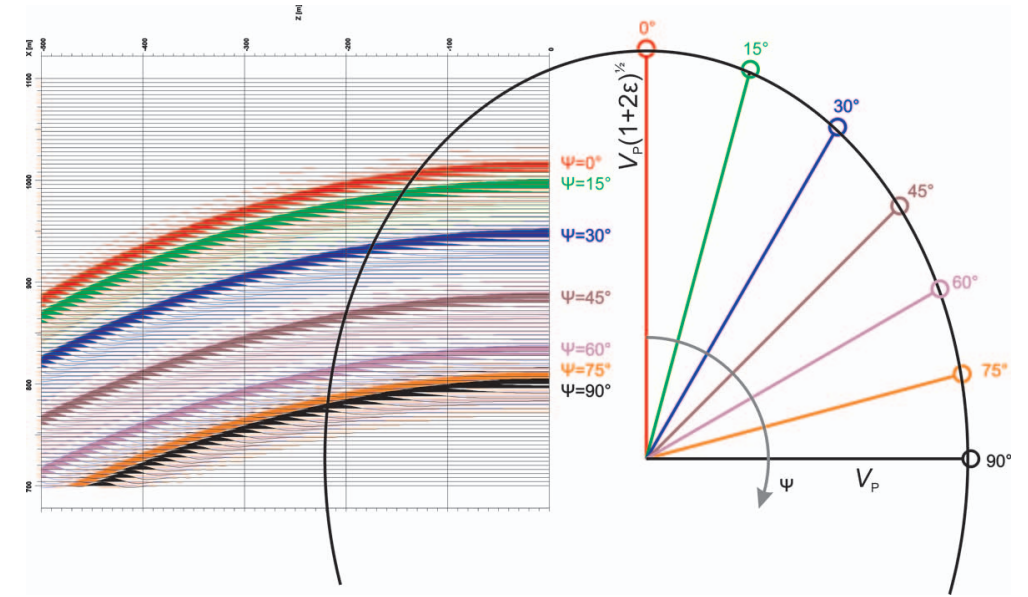

Fig. 9. Velocity as a function of the azimuthal angle $\Psi$ in the first quarter of the clockwise polar coordinate system. The ellipsoid character of velocity $(\Psi)$ is seen. In the left part of the figure we have a series of wavefronts which determine the length of propagation paths for time $t=0.2 \mathrm{~s}, \varepsilon=0.3 ; \delta=0.1$

\section{Modeling and migration of zero-offset time sections}

The subject of numerical experiments was the inhomogeneous four layers model of anticline, covered by a horizontal layer of thickness $500 \mathrm{~m}$ (Fig. 10). The velocity of compressional waves, constant in each layer, changes from $3.0 \mathrm{~km} / \mathrm{s}$ to $5.0 \mathrm{~km} / \mathrm{s}$.

The wavefield for this zero-offset time section was computed by a simultaneously exited reflectors method at $t=0$ [23]. A pseudo-spectral version of one-way equation was used (6) and (11) in view of lateral inhomogeneity (14). A part of the comprehensive experiment of modeling is presented in Fig. 11-14 for anisotropy parameters: $\varepsilon=0.3 ; \delta=0.1$ and azimuthal angles: $\Psi=15^{\circ}, 45^{\circ}$, $75^{\circ}, 90^{\circ}$. Analyzing the time sections, one can note that for increasing azimuthal angles, curves of runtimes decrease, whereas summits of anticline change only a little, mainly displacing in a lateral direction. In light of the analyses of propagation results in dependence on azimuthal angles $\Psi$ (Fig. 8-9) this occurrence is fully justified by maximum velocity $V=V_{p}(1+2 \varepsilon)^{1 / 2}$ in vertical direction and by a decrease of velocity in lateral direction.
Depth mappings made with the use of zero-offset migration (Fig. 15-18) demonstrate practically invariable images for various azimuthal angles, as expected.

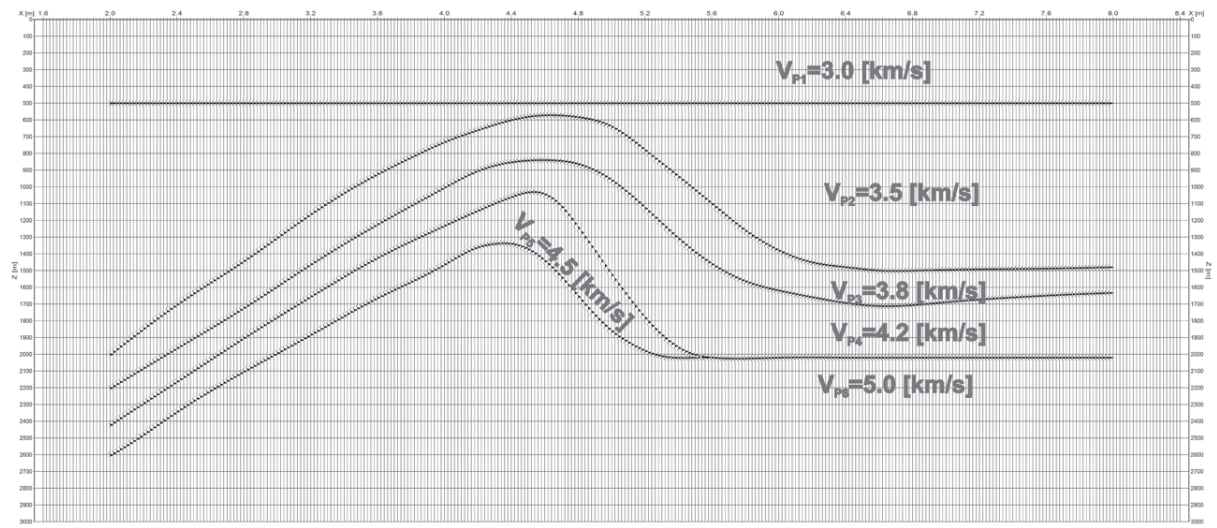

Fig. 10. The geometry of velocity model

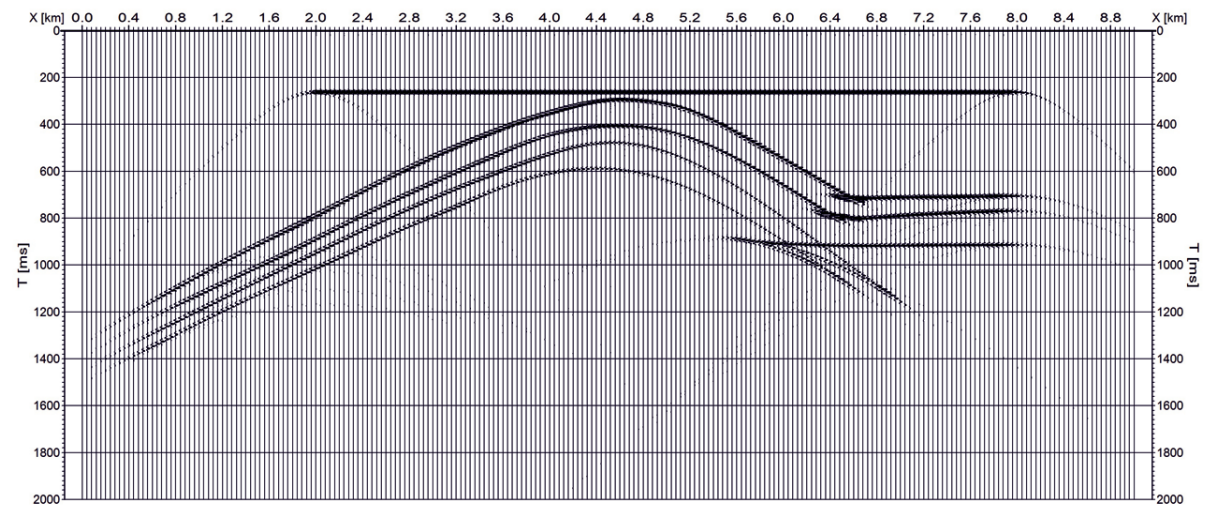

Fig. 11. The zero-offset time section from relations (6); anisotropy parameters $\varepsilon=0.3 ; \delta=0.1$; azimuthal angle $\Psi=15^{\circ}$ 


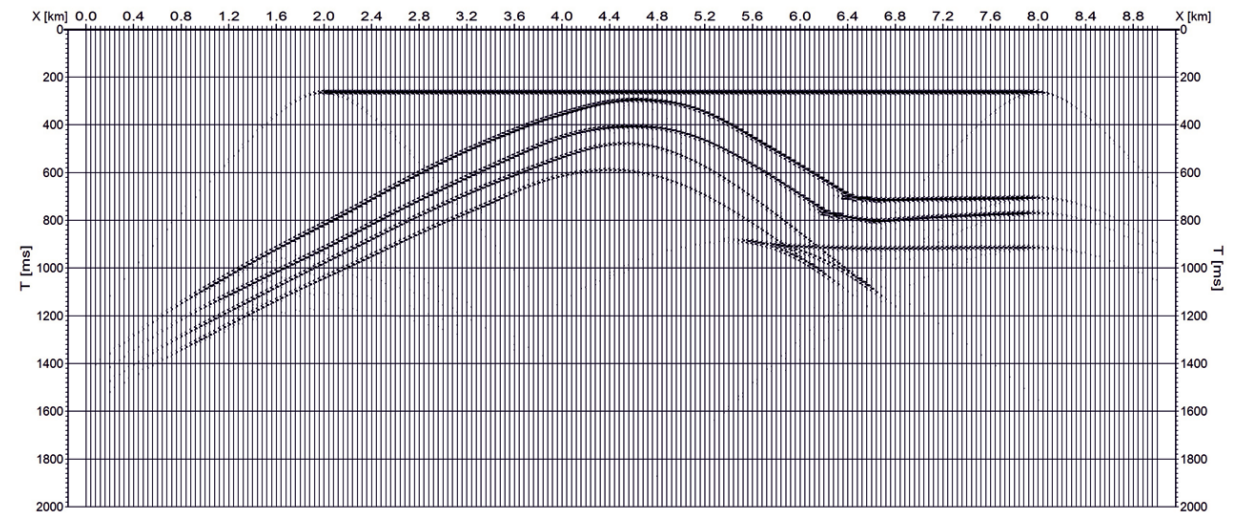

Fig. 12. The zero-offset time section from relations (6); anisotropy parameters $\varepsilon=0.3 ; \delta=0.1$; azimuthal angle $\Psi=45^{\circ}$

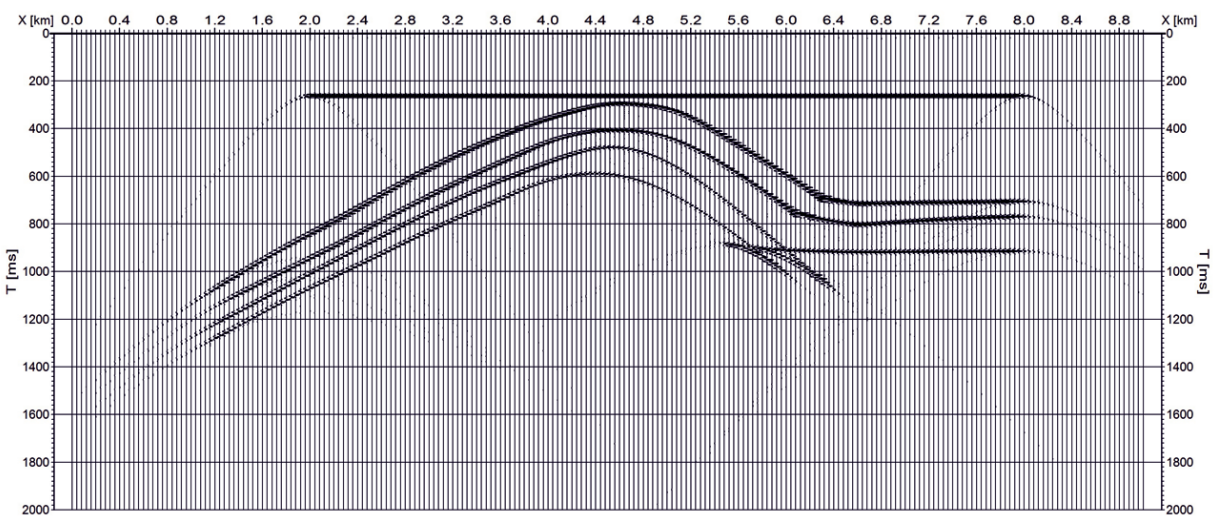

Fig. 13. The zero-offset time section from relations (6); anisotropy parameters $\varepsilon=0.3 ; \delta=0.1$; azimuthal angle $\Psi=75^{\circ}$

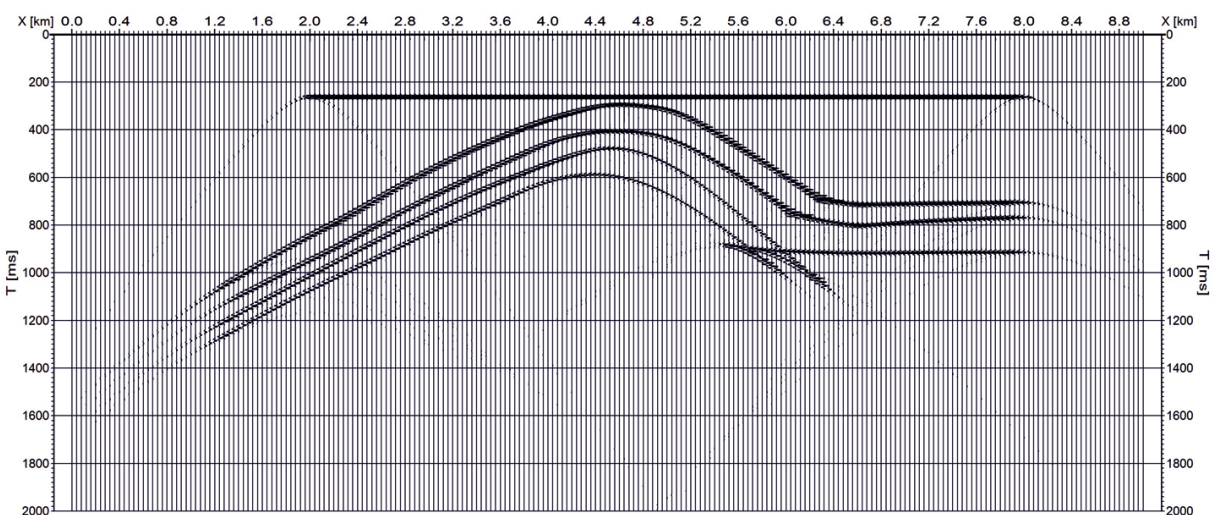

Fig. 14. The zero-offset time section from relations (6); anisotropy parameters $\varepsilon=0.3 ; \delta=0.1$; azimuthal angle $\Psi=90^{\circ}$

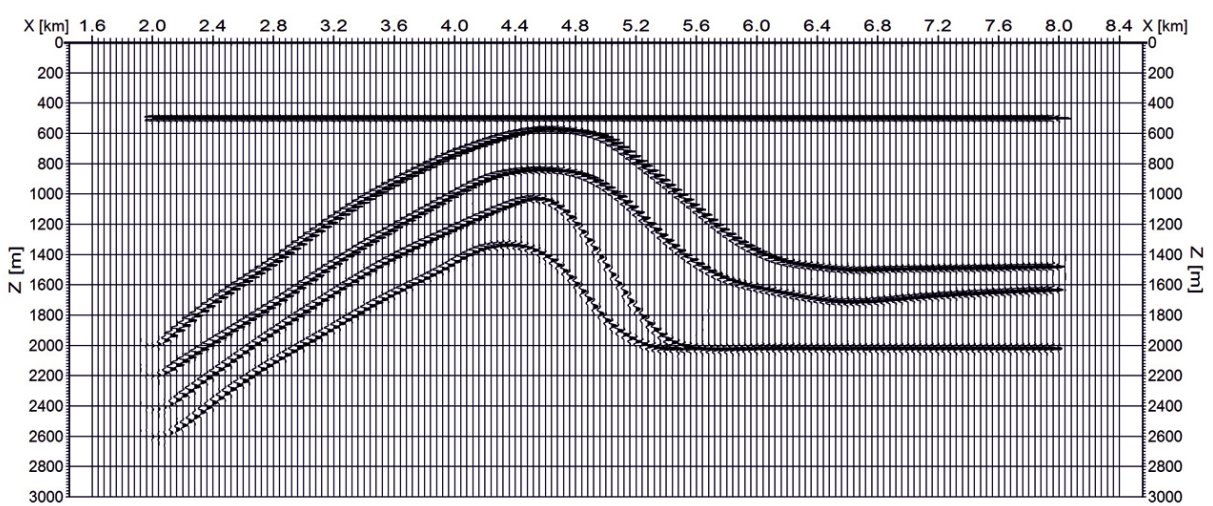

Fig. 15. The migration according to (28) for the zero-offset time section Fig. 11 (relation 6); anisotropy parameters $\varepsilon=0.3 ; \delta=0.1$; azimuthal angle $\Psi=15^{\circ}$ 


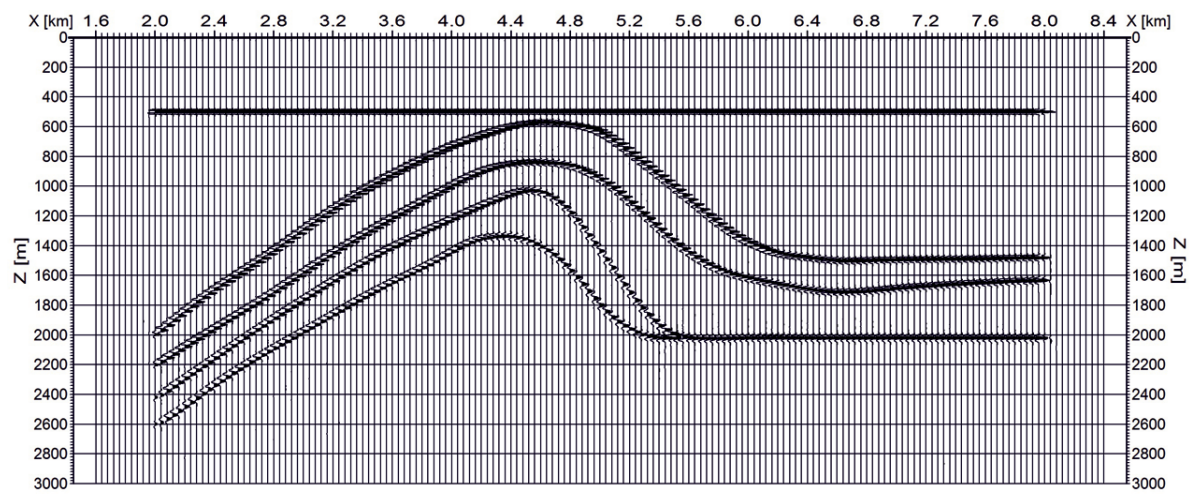

Fig. 16. The migration according to (28) for the zero-offset time section Fig. 12 (relation 6); anisotropy parameters $\varepsilon=0.3 ; \delta=0.1$; azimuthal angle $\Psi=45^{\circ}$

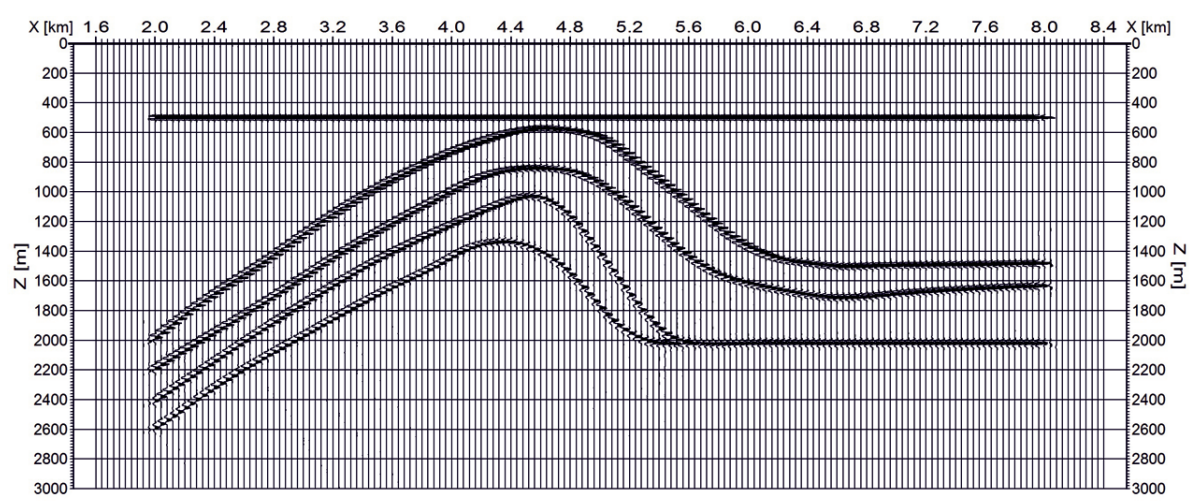

Fig. 17. The migration according to (28) for the zero-offset time section Fig. 13 (relation 6); anisotropy parameters $\varepsilon=0.3 ; \delta=0.1$; azimuthal angle $\Psi=75^{\circ}$

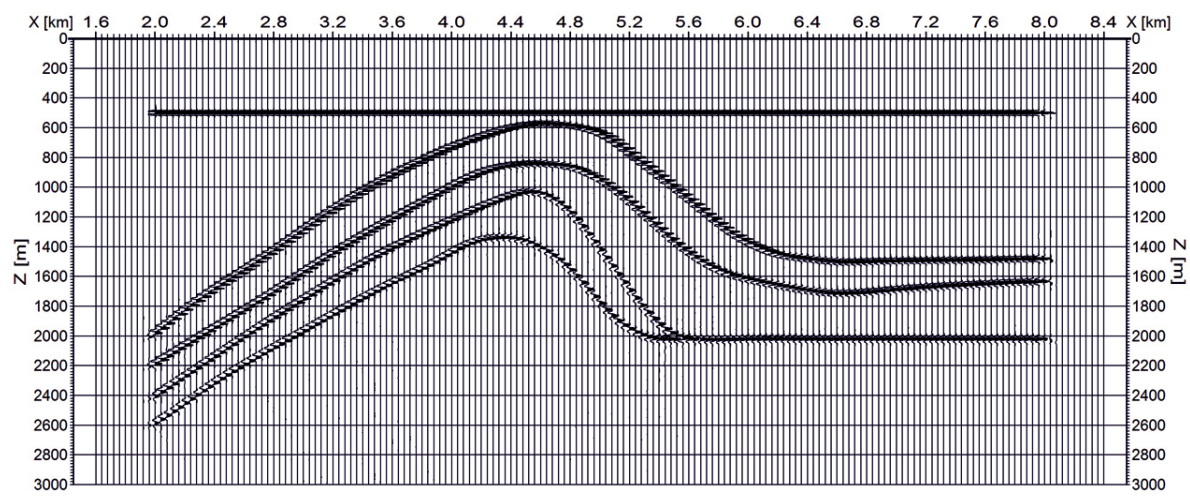

Fig. 18. The migration according to (28) for the zero-offset time section Fig. 14 (relation 6); anisotropy parameters $\varepsilon=0.3 ; \delta=0.1$; azimuthal angle $\Psi=90^{\circ}$

\section{Conclusion}

We have presented new ways of wave propagation in forward and in backward projection, i.e. seismic migration in anisotropic media of the HTI type in the function of azimuthal angle $\Psi$.

Forward modeling is described by pseudo-acoustic equations (in one-way or in two-sided versions) in wavenumber and time-frequency domain derived as eigenvalues from the dispersion relation.
This way of modeling is devoid of the "diamond shape" type noise, characteristic for pseudo-acoustic equations in space coordinates and enables to reach high accuracy (with error $\approx 0.2 \%$ for time $0.4 \mathrm{~s}$ ). The forward modeling and "back propagation" as well, i.e. migration are based on dispersion relation derived from the full system of elasticity equations. In case of migration it is necessary to calculate vertical wavenumbers as function of time, frequency, wavenumbers, 
Thomsen's anisotropy parameters: $\varepsilon, \delta$ and azimuthal angle $\Psi$ to determine the depth operator of extrapolation.
Examples of post-stack migration made on an anisotropic model of an anticline, demonstrate the efficiency of imaging.

Please cite as: Nafta-Gaz 2016, no. 9, pp. 679-690, DOI: 10.18668/NG.2016.09.01

Article contributed to the Editor 20.04.2016. Approved for publication 10.06.2016.

The article is the result of research conducted in connection with the project: Seismic tests and their application in detection of shale gas zones. Selection of optimal parameters for acquisition and processing in order to reproduce the structure and distribution of petrophysical and geomechanical parameters of prospective rocks, as part of the programme BLUE GAS POLISH SHALE GAS. Contract No. BG1/GASLUPSEJSM/13.

\section{Literature}

[1] Alkhalifah T.: Acoustic approximation for processing in transversely isotropic media. Geophysics 1998, vol. 63, no. 2, pp. 623-631.

[2] Alkhalifah T.: An acoustic wave equation for anisotropic media. Geophysics 2000, vol. 65, no. 4, pp. 1239-1250.

[3] Alkhalifah T.: Traveltime computation with the linearized eikonal equation for anisotropic media. Geophysical Prospecting 2002, vol. 50, pp. 373-382.

[4] Auld B.: Acoustic field and waves in solid. Krieger Publishing Company 1990, vol. 1.

[5] Baysal E., Kosloff D. D., Sherwood J. W.: Reverse time migration. Geophysics 1984, vol. 48, no. 9, pp.1411-1419.

[6] Bube K., Nemeth T., Stefani J., Ergas R., Wei Liu, Nihei K., Zhang L.: On the instability in second-order systems for acoustic VTI and TTI media. Geophysics 2012, vol. 77, no. 5, pp. T171-T186.

[7] Bube K., Nemeth T., Stefani J., Wei Liu, Nihei K., Ergas R., Zhang L.: First-order systems of elastic and acoustic variable-tilt TI media. Geophysics 2012, vol. 77, no. 5, pp. T157-T170.

[8] Cerveny W.: Seismic ray theory. Cambridge University Press 2001.

[9] Du X., Fletcher R., Fowler P. J.: A new pseudo-acoustic wave equation for TI media. $70^{\text {th }}$ Annual International Conference and Exhibition, EAGE 2008, Extended Abstracts, H033.

[10] Duveneck E., Bakker P. M.: Stable P-wave modeling for reverse time migration in tilted media. Geophysics 2011, vol. 76 , no. 2 , pp. 565-575, doi: 10.1190/1.3533964.

[11] Faria E., Stoffa P.: Traveltime computation in transversely isotropic media. Geophysics 1994, vol. 59, pp. 272-281.

[12] Fletcher R., Du X., Fowler P. J.: A new pseudo-acoustic wave equation for TI media. Annual Meeting, Las Vegas, SEG 2008, Extended Abstracts

[13] Gajewski D., Pŝencik V.: Computation of high-frequency seismic wavefield in 3D-laterally inhomogeneous anisotropic media. Geophysical Journal of the Royal Astronomical Society 1987, vol. 91, pp. 381-411.

[14] Gazdag I.: Modeling of the acoustic wave equation with transform methods. Geophysics 1981, vol. 46, pp. 854-859.

[15] Grechka V., Tsvankin I.: 3D description of normal moveout in anisotropic inhomogeneous media. Geophysics 1998, vol. 63, pp. 1079-1092.

[16] Kostecki A.: Algorithm of prestack migration of wavefield. Proc. First Science-Tech. Conf. The Seismic Problems of the Interpretation, Cracow, Poland 1994.

[17] Kostecki A.: Tilted Transverse Isotropy. Nafta-Gaz 2011, no. 11 pp. 769-776.

[18] Kostecki A., Półchłopek A., Żuławiński K.: The imaging structures

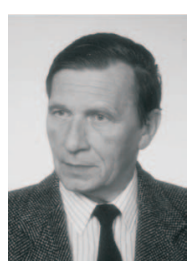

Prof. dr hab. Eng. Andrzej Kostecki

Full Professor

Oil and Gas Institute - National Research Institute

ul. Lubicz 25 A

31-503 Kraków

E-mail: andrzej.kostecki@inig.pl in anisotrpic media by seismic migration. Oil and Gas Institute, monography 2013.

[19] Kostecki A., Półchłopek A.: Stable depth extrapolation of seismic wavefields by a Neumann series. Geophysics 1998, vol. 63, no. 6, pp. 2063-2071.

[20] Kostecki A., Zuławiński K.: Modeling and migration of zero-offset time sections in TTI media by pseudo-spectral method. Nafta-Gaz 2014, no. 12, pp. 855-860.

[21] Kostecki A., Żuławiński K.: The pseudo-acoustic equations of the scalar wavefield in anisotropic media. Nafta-Gaz 2015, no. 11, pp. 811-815.

[22] Kumar D., Sen M., Ferguson R.: Traveltime calculation and prestack migration in tilted transversely isotropic media. Geophysics 2004, vol. 69, pp. 37-44. doi: 10.1190/1.1649373.

[23] Loewenthal D., Lu L., Robertson R., Sherwod I.: The wave equation applied to migration. Geophysical Prospecting 1976, vol. 24, no. 2, pp. 380-399.

[24] Mc Mechan G. A.: Migration by extrapolation of time dependent boundary values. Geophysical Prospecting 1983. vol. 31, no. 4 pp. 413-420.

[25] Podvin P., Lecomte I.: Finite difference computation of traveltimes in very contrasted velocity models. A massively parallel approach and its associated tools. Geophysical Journal International 1991, vol. 105 , pp. 272-284.

[26] Schneider W. A.: Integral formulation for migration in two and three dimensions. Geophysics 1978, vol. 43, no. 1, pp. 49-76.

[27] Sena A., Töksoz M. N.: Kirchoff migration and velocity analysis for converted and nonconverted waves in anisotropic media. Geophysics 1993, vol. 58, pp. 265-276.

[28] Shearer P., Chapman C.: Ray tracing in anisotropic media with linear gradient. Geoph. Inter. 1988, vol. 94, pp. 575-580.

[29] Tsvankin I., Grechka V.: Seismology of azimuthally anisotropic media and seismic fracture characterization. Geophysical Reference Series 17: SEG, 2011.

[30] Xie W., Yang D., Liu F., Li J.: Reverse - time migration in acoustic VTI media using a high-order stereo - operator. Geophysics 2014 vol. 79, no. 3, pp. WA3-WA13.

[31] Zhang L., Rector III J. W., Hoversten M.: Finite-difference modeling of wave propagation in acoustic tilted TI media. Geophysical Prospecting 2005, vol. 53, pp. 843-852.

[32] Zhu J., Dorman J.: Two-dimensional, three-component wave propagation in a transversely isotropic medium with arbitrary-orientationfinite element modeling. Geophysics 2000, vol. 65, pp. 934-942.

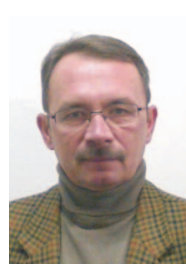

Krzysztof ŻUŁAWIŃSKI M.Sc. Eng.

Senior Technical Research Specialist

Seismic Department

Oil and Gas Institute - National Research Institute

ul. Lubicz 25 A

31-503 Kraków

E-mail: krzysztof.zulawinski@inig.pl 


\section{Appendix}

The tensor components as the a function of dip angle $\theta($ for $+\theta)$ and azimuthal angle $\Psi$ are as follow:

$d_{11}^{\theta, \Psi}=C_{11}\left[\cos ^{4} \theta \sin ^{4} \Psi+\cos ^{4} \Psi+\frac{1}{2} \cos ^{2} \theta \sin ^{2}(2 \Psi)\right]+C_{33} \sin ^{4} \theta \sin ^{4} \Psi+\frac{1}{2} C_{13}\left[\sin ^{2} \theta \sin ^{2}(2 \Psi)+\sin ^{2}(2 \theta) \sin ^{4} \Psi\right]+$ $+C_{44}\left[\sin ^{2} \theta \sin ^{2}(2 \Psi)+\sin ^{4} \Psi \sin ^{2}(2 \theta)\right]$

$\begin{aligned} d_{12}^{\theta \Psi}= & C_{11}^{\theta \Psi}\left\{\cos ^{2} \theta\left[\sin ^{4} \Psi+\cos ^{4} \Psi+\frac{1}{4} \cos ^{2} \theta \sin ^{2}(2 \Psi)\right]+\frac{1}{4} \sin ^{2}(2 \Psi)\right\}+C_{13}\left[\frac{1}{8} \sin ^{2}(2 \theta) \sin ^{2}(2 \Psi)+\sin ^{2} \theta\left(\sin ^{4} \Psi+\cos ^{4} \Psi\right)\right]+ \\ & +\frac{1}{4} C_{33} \sin ^{4} \theta \sin ^{2}(2 \Psi)+C_{44}\left[\frac{1}{4} \sin ^{2}(2 \theta) \sin ^{2}(2 \Psi)-\sin ^{2} \theta \sin ^{2}(2 \Psi)\right]-C_{66} \cos ^{2} \theta\left[\sin ^{2}(2 \Psi)+2\left(\sin ^{4} \Psi+\cos ^{4} \Psi\right)\right]\end{aligned}$

$\begin{aligned} & d_{13}^{\theta, \Psi} \\ & d_{31}^{\theta, \Psi}\end{aligned}=C_{11}\left[\frac{1}{4} \sin ^{2}(2 \theta) \sin ^{2} \Psi+\sin ^{2} \theta \cos ^{2} \Psi\right]+C_{13}\left[\sin ^{4} \theta \sin ^{2} \Psi+\cos ^{4} \theta \sin ^{2} \Psi+\cos ^{2} \theta \cos ^{2} \Psi\right]+$ $+\left(\frac{1}{4} C_{33}-C_{44}\right) \sin ^{2}(2 \theta) \sin ^{2} \Psi-2 C_{66} \sin ^{2} \theta \cos ^{2} \Psi$

$\begin{aligned} & d_{14}^{\theta \Psi} \\ & d_{41}^{\theta \Psi}\end{aligned}=\frac{1}{2}\left(C_{13}-C_{11}\right) \sin (2 \theta)\left[\frac{1}{2} \cos ^{2} \theta \sin \Psi \sin (2 \Psi)+\cos ^{3} \Psi\right]+\frac{1}{4}\left(C_{33}-C_{13}\right) \sin (2 \theta) \sin (2 \Psi) \sin ^{2} \theta \sin \Psi-$ $-\frac{1}{2} C_{44} \sin (2 \theta) \sin (2 \Psi) \sin \Psi[1-\cos (2 \theta)]+C_{66} \sin (2 \theta)\left[\frac{1}{2} \sin (2 \Psi) \sin \Psi+\cos ^{3} \Psi\right]$

$d_{15}^{\theta, \Psi}$
$d_{51}^{\theta, \Psi}=\frac{1}{2}\left(C_{11}-C_{13}\right) \sin (2 \theta) \sin \Psi\left[\cos ^{2} \theta \sin ^{2} \Psi+\cos ^{2} \Psi\right]+\frac{1}{2}\left(C_{13}-C_{33}\right) \sin (2 \theta) \sin ^{2} \theta \sin ^{3} \Psi-$ $-\frac{1}{2} C_{44}\left[\sin (2 \theta) \sin (2 \Psi) \cos \Psi+\sin (4 \theta) \sin ^{3} \Psi\right]$

$d_{44}^{\theta, \Psi}=\frac{1}{4}\left(C_{11}-2 C_{13}+C_{33}\right) \cos ^{2} \Psi \sin ^{2}(2 \theta)+C_{44}\left[\cos ^{2} \theta \sin ^{2} \Psi+\cos ^{2} \Psi \cos ^{2}(2 \theta)\right]+C_{66} \sin ^{2} \theta \sin ^{2} \Psi$ $\begin{aligned} & d_{45}^{\theta, \Psi} \\ & d_{54}^{\theta, \Psi}\end{aligned}=-\frac{1}{8}\left(C_{11}-2 C_{13}+C_{33}\right) \sin (2 \Psi) \sin ^{2}(2 \theta)+\frac{1}{2} C_{44} \sin (2 \Psi)\left[\cos ^{2} \theta-\cos ^{2}(2 \theta)\right]+\frac{1}{2} C_{66} \sin (2 \Psi) \sin ^{2} \theta$ $\underset{d_{64}^{\theta, \Psi}}{d_{46}^{\theta \Psi}}=\frac{1}{4}\left(2 C_{13}-C_{11}-C_{33}\right) \sin (2 \Psi) \sin (2 \theta) \cos \Psi \sin ^{2} \theta+\frac{1}{2} C_{44} \sin (2 \theta)[\sin \Psi \cos (2 \Psi)-\sin (2 \Psi) \cos (2 \theta) \cos \Psi]+$ $+\frac{1}{2} C_{66} \sin (2 \theta)[\cos \Psi \sin (2 \Psi)-\sin \Psi \cos (2 \Psi)]$

$d_{55}^{\theta, \Psi}=\frac{1}{4}\left(C_{11}-2 C_{13}+C_{33}\right) \sin ^{2} \Psi \sin ^{2}(2 \theta)+C_{44}\left[\cos ^{2} \theta \cos ^{2} \Psi+\sin ^{2} \Psi \cos ^{2}(2 \theta)\right]+C_{66} \sin ^{2} \theta \cos ^{2} \Psi$ $d_{56}^{\theta, \Psi}=\frac{1}{4}\left(C_{11}-2 C_{13}+C_{33}\right) \sin (2 \Psi) \sin (2 \theta) \sin \Psi \sin ^{2} \theta+\frac{1}{2} C_{44} \sin (2 \theta)[\cos \Psi \cos (2 \Psi)+\sin (2 \Psi) \cos (2 \theta) \sin \Psi]-$ $-\frac{1}{2} C_{66} \sin (2 \theta)[\cos \Psi \cos (2 \Psi)+\sin \Psi \sin (2 \Psi)]$ 


\section{NAFTA-GAZ}

$d_{66}^{\theta, \Psi}=\frac{1}{4}\left(C_{11}-2 C_{13}+C_{33}\right) \sin ^{2}(2 \Psi) \sin ^{4} \theta+C_{44}\left[\sin ^{2} \theta \cos ^{2}(2 \theta)+\frac{1}{4} \sin ^{2}(2 \theta) \sin ^{2}(2 \Psi)\right]+C_{66} \cos ^{2} \theta$

$\begin{aligned} & d_{16}^{\theta, \Psi} \\ & d_{61}^{\theta, \Psi}\end{aligned}=\frac{1}{2} C_{11} \sin (2 \Psi)\left[\cos ^{2} \Psi-\cos ^{2} \theta \cos (2 \Psi)-\cos ^{4} \theta \sin ^{2} \Psi\right]-\frac{1}{2} C_{33} \sin ^{4} \theta \sin ^{2} \Psi \sin (2 \Psi)-$

$-\left(\frac{1}{4} C_{13}+\frac{1}{2} C_{44}\right)\left[\sin (4 \Psi) \sin ^{2} \theta+\sin ^{2}(2 \theta) \sin (2 \Psi) \sin ^{2} \Psi\right]$

$d_{22}^{\theta, \Psi}=C_{11}\left[\cos ^{4} \theta \cos ^{4} \Psi+\frac{1}{2} \cos ^{2} \theta \sin ^{2}(2 \Psi)+\sin ^{4} \Psi\right]+\frac{1}{2} C_{13}\left[\sin ^{2}(2 \theta) \cos ^{4} \Psi+\sin ^{2} \theta \sin ^{2}(2 \Psi)\right]+$

$+C_{33} \sin ^{4} \theta \cos ^{4} \Psi+C_{44}\left[\sin ^{2} \theta \sin ^{2}(2 \Psi)+\sin ^{2}(2 \theta) \cos ^{4} \Psi\right]$

$\begin{aligned} & d_{23}^{\theta \Psi} \\ & d_{32}^{\theta \Psi}\end{aligned}=C_{11} \sin ^{2} \theta\left(\cos ^{2} \theta \cos ^{2} \Psi+\sin ^{2} \Psi\right)+C_{13}\left[\cos ^{2} \theta\left(\cos ^{2} \theta \cos ^{2} \Psi+\sin ^{2} \Psi\right)+\sin ^{4} \theta \cos ^{2} \Psi\right]_{+}$

$+C_{33} \cos ^{2} \theta \sin ^{2} \theta \cos ^{2} \Psi-C_{44} \sin ^{2}(2 \theta) \cos ^{2} \Psi-2 C_{66} \sin ^{2} \theta \sin ^{2} \Psi$

$\begin{aligned} & d_{24}^{\theta, \Psi} \\ & d_{42}^{\theta, \Psi}\end{aligned}=\frac{1}{2}\left(C_{13}-C_{11}\right) \cos \Psi \sin (2 \theta)\left(\cos ^{2} \Psi \cos ^{2} \theta+\sin ^{2} \Psi\right)+\frac{1}{2}\left(C_{33}-C_{13}\right) \cos ^{3} \Psi \sin ^{2} \theta \sin (2 \theta)+$

$+\frac{1}{2} C_{44}\left[\sin (2 \theta) \sin (2 \Psi) \sin \Psi+\cos ^{3} \Psi \sin (4 \theta)\right]$

$\begin{aligned} & d_{25}^{\theta, \Psi} \\ & d_{52}^{\theta, \Psi}\end{aligned}=\frac{1}{2}\left(C_{11}-C_{13}\right) \sin \Psi \sin (2 \theta)\left(\cos ^{2} \Psi \cos ^{2} \theta+\sin ^{2} \Psi\right)+\frac{1}{2}\left(C_{13}-C_{33}\right) \sin \Psi \sin (2 \theta) \sin ^{2} \theta \cos ^{2} \Psi+$

$+\frac{1}{2} C_{44} \sin (2 \Psi) \sin (2 \theta) \cos \Psi[1-\cos (2 \theta)]-C_{66} \sin (2 \theta)\left[\frac{1}{2} \sin (2 \Psi) \cos \Psi+\sin ^{3} \Psi\right]$

$\begin{aligned} & d_{26}^{\theta, \Psi} \\ & d_{62}^{\theta, \Psi}\end{aligned}=\frac{1}{2} C_{11} \sin (2 \Psi) \sin ^{2} \theta\left(\cos ^{2} \Psi \cos ^{2} \theta+\sin ^{2} \Psi\right)+\frac{1}{2} C_{13} \sin (2 \Psi) \sin ^{2} \theta\left[\cos (2 \Psi)-2 \cos ^{2} \theta \cos ^{2} \Psi\right]-$

$-\frac{1}{2} C_{33} \sin ^{4} \theta \cos ^{2} \Psi \sin (2 \Psi)+\frac{1}{2} C_{44}\left[\sin ^{2} \theta \sin (4 \Psi)-\sin ^{2}(2 \theta) \cos ^{2} \Psi \sin (2 \Psi)\right]$

$d_{33}^{\theta, \Psi}=C_{11} \sin ^{4} \theta+2 C_{13} \sin ^{2} \theta \cos ^{2} \theta+C_{33} \cos ^{4} \theta+C_{44} \sin ^{2}(2 \theta)$

$\begin{aligned} & d_{34}^{\theta, \Psi} \\ & d_{43}^{\theta, \Psi}\end{aligned}=\frac{1}{2}\left(C_{33} \cos ^{2} \theta-C_{11} \sin ^{2} \theta\right) \cos \Psi \sin (2 \theta)-\left(\frac{1}{4} C_{13}+\frac{1}{2} C_{44}\right) \cos \Psi \sin (4 \theta)$

$d_{35}^{\theta, \Psi}=\frac{1}{2}\left[\left(C_{11}-C_{13}\right) \sin ^{2} \theta+\left(C_{13}-C_{33}\right) \cos ^{2} \theta\right] \sin \Psi \sin (2 \theta)+\frac{1}{2} C_{44} \sin \Psi \sin (4 \theta)$

$d_{36}^{\theta \Psi \Psi}=\frac{1}{2}\left(C_{11}-C_{13}\right) \sin (2 \Psi) \sin ^{4} \theta+\frac{1}{2}\left(C_{13}-C_{33}\right) \sin (2 \Psi) \sin ^{2} \theta \cos ^{2} \theta+\frac{1}{2} C_{44} \sin (2 \Psi) \sin ^{2}(2 \theta)-C_{66} \sin (2 \Psi) \sin ^{2} \theta$ 